\title{
PHOTOINDUCED ELECTRON TRANSFER IN JET COOLED MOLECULAR COMPLEXES
}

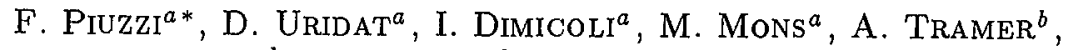 \\ K. LeBarbu ${ }^{b}, F$. LAHMANi ${ }^{b}$ and A. ZeHNACKeR-Rentien ${ }^{b}$ \\ ${ }^{a}$ CEA-CEN Saclay, DRECAM, SPAM, Bat 522, 91191, Gif sur Yvette Cedex, France \\ ${ }^{b}$ Laboratoire de Photophysique Moléculaire du CNRS \\ Bat 213, Université de Paris Sud, 91405 Orsay, France
}

\begin{abstract}
Exciplex and excimer formation have been probed in several jet cooled complexes using mass selective two-photon ionisation and fluorescence excitation spectroscopy as well as ground state depletion spectroscopy (hole burning): (i) In the anthracene-dimethyl-ortho-toluidine system, it has been found that the ionisation step takes place with a much higher efficiency from the charge transfer state responsible for the exciplex emission than from the locally excited state giving rise to the resonant fluorescence. (ii) The dimer, trimer, and higher clusters of anthracene all show only excimer emission. When compared to the dimer, the trimer exhibits a peculiar behaviour (structured fluorescence excitation and hole burning spectra, short lifetime and low ionisation efficiency) which has been related to a significant locally excited character of the initially prepared state of the species excited state. (iii) The influence of an intermolecular hydrogen bond on the electron transfer process has been studied in the 2,2,2-trifluoro-1-(9-anthryl)ethanoldimethylaniline system. A threshold for exciplex formation higher than in the case of the anthracene-dimethylaniline complex is observed.
\end{abstract}

PACS numbers: 31.50.+w, 32.30.-r, 33.15.-e, 33.20.-t

\section{Introduction}

In previous papers [1] we reported studies concerning photoinduced electron transfer in jet-cooled acceptor-donor (AD) complexes of anthracene (An) with $N, N$-dimethylaniline (DMA) and its analogues: $o$-, $m$ - and $p-N, N$-dimethyltoluidine (DMOT, DMMT and DMPT) as well as $N, N$-diethylaniline (DEA). All complexes display a rich collection of isomeric forms: from two for An-DMA case to six in that of An-DMOT. The fluorescence and fluorescence excitation spectra have been recorded for all of them while the hole burning spectroscopy [2] allowed us to separate spectral features belonging to each isomeric form. Depending on the selected isomeric form different behaviours with respect to the electron transfer process have been evidenced and the general conclusions from this work may be summarised in the following way:

* Corresponding author. 
(1) The fluorescence excitation spectra of all forms are red shifted by ca. 400 to $700 \mathrm{~cm}^{-1}$ with respect to the absorption of bare anthracene and reproduce its vibrational structure involving the $\nu_{11}^{\prime}=232 \mathrm{~cm}^{-1}$ and $\nu_{12}^{\prime}=385 \mathrm{~cm}^{-1}$ modes. in Fig. 1):

(2) The fluorescence spectra consist of two well separated components (scheme

(i) a narrow band emission involving the optically active anthracene modes and low frequency intermolecular modes with the lifetime of 20 to $25 \mathrm{~ns}$, close to that of the free anthracene - assigned to the resonant fluorescence from the $A^{*} D$ van der Waals state,

(ii) strongly red shifted, diffuse exciplex fluorescence with a long (250 to $300 \mathrm{~ns}$ ) lifetime corresponding to the emission from the ionic $\mathrm{A}^{-} \mathrm{D}^{+}$state.

(3) Two different types of fluorescence excitation spectra (FES) which correspond to two types of isomers are observed:

(i) the $\mathrm{E}$ type isomers exhibit FES composed of relatively broad $\left(\approx 100 \mathrm{~cm}^{-1}\right)$ $0_{0}^{0}$ and $12_{0}^{1}$ bands present only in the excitation spectra of exciplex fluorescence (E-FES). This emission indicates that the $\mathrm{A}^{*} \mathrm{D} \leadsto \mathrm{A}^{-} \mathrm{D}^{+}$transition takes place even from the lowest levels of the initially prepared $\mathrm{A}^{*} \mathrm{D}$ state at the time scale much shorter than the fluorescence lifetime of the $A^{*} D$ state $\left(1 / \tau>2 \times 10^{12} \mathrm{~s}^{-1}\right.$ as estimated from the band widths),

(ii) the $\mathrm{R}$ type isomers display FES composed of narrow bands involving intermolecular modes accompanying each of $0_{0}^{0}$ and $122_{0}^{1}$ transitions. For most systems, the resonant emission is observed upon the excitation of the $0_{0}^{0}$ band system, thus appearing in the resonant fluorescence excitation spectrum (R-FES) while the excitation of the $12_{0}^{1}$ one yields only the exciplex fluorescence which implies that it appears in the E-FES. Obviously, the $\mathrm{A}^{*} \mathrm{D} \leadsto \mathrm{A}^{-} \mathrm{D}^{+}$transition takes place only from higher levels of the $A^{*} D$ state (its onset typically contained in the 100 to $220 \mathrm{~cm}^{-1}$ limit) and is relatively slow $\left(1 / \tau<10^{11} \mathrm{~s}^{-1}\right)$.

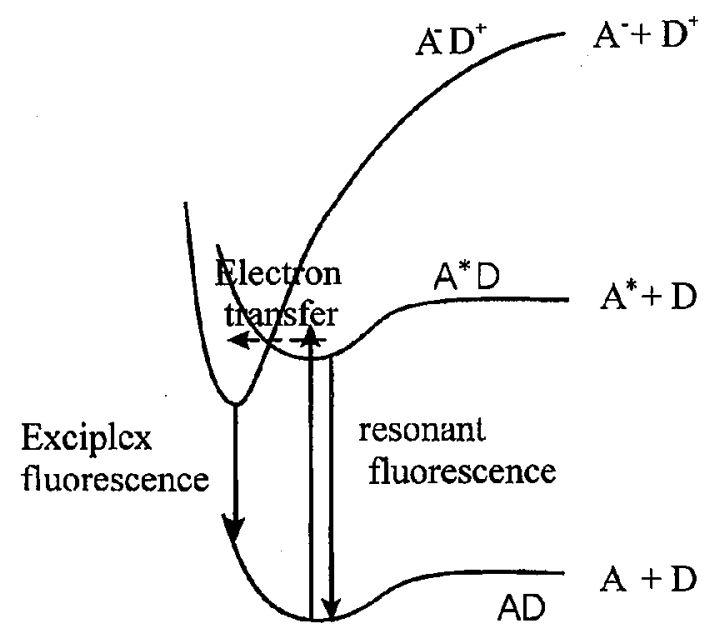

Fig. 1. Fundamental parameters of photoinduced electron transfer in acceptor-donor complexes. 
We present here new results on jet cooled $\mathrm{AD}$ and related systems which enable to get further information on the electron transfer process in isolated systems.

(1) Ionization of photoexcited $A D$ van der Waals complexes.

The lack of selectivity of the spectroscopic methods based on fluorescence detection leads in many cases to difficulties in the interpretation of the results since many species which may fluoresce are formed in the expansion. The use of mass selective spectroscopic methods is then important in order to unambiguously determine which species correspond to the different features of the fluorescence excitation spectra (provided that all the species are efficiently ionised). However the observation of differences between FES and mass selective excitation spectra (MSES) may also give interesting information about the nature and the dynamics of the species under study. This method will be applied to the case of the An-DMOT complex.

(2) Excimer formation from photoexcited anthracene dimers and clusters.

Excitation of dimers or clusters of different aromatic compounds like naphthalene $[3,4]$, dibenzofuran $[5,6]$ or anthracene [7] results in a broad structureless and strongly red shifted emission. This so-called excimer emission is the analogue of the exciplex emission observed after photoexcitation of AD complexes. Thus it is instructive to compare the anthracene dimer properties to that of $\mathrm{AD}$ complexes: rate and threshold of excimer formation, dependence of the electron transfer process on the configuration.

(3) Electron transfer in AD complexes bonded by a hydrogen bond.

In order to investigate the role of the structural factors that can modify the electron transfer, we are currently studying jet-cooled complexes between a chiral anthracene derivative, 2,2,2-trifluoro-1-(9-anthryl)ethanol (TFAE), as an electron acceptor and an aniline derivative as a donor. In these complexes, the geometry is expected to be constrained by a strong interaction related to the presence of a hydrogen bond between the acceptor and the donor. On the other hand, TFAE is known as a chiral resolving agent for chiral phase chromatography: it has been used to discriminate between enantiomers of amines [8], so the study of the nature of the interactions between TFAE and amines can provide information about the nature of the forces responsible for chiral discrimination in these systems.

\section{Experimental}

All experiments are performed in a pulsed supersonic expansion using a General Valve nozzle and helium as a carrier gas. The vacuum system is composed of two chambers separated by a skimmer. The laser beams cross the supersonic jet in the first chamber, where the fluorescence is detected through a $20 \mathrm{~cm}$ Bausch and Lomb monochromator acting as a broad-band band-pass filter (375 $\mathrm{nm}$ for detection of the resonant fluorescence or at $450 \mathrm{~nm}$ for the exciplex/excimer fluorescence). The beams are issued from two Lambda Physik FL 2002 dye laser with a $c a .0 .2 \mathrm{~cm}^{-1}$ linewidth pumped by one (or two when a large delay is needed) Lambda Physik excimer lasers. For the hole burning experiment, we used a low intensity $(100-200 \mu \mathrm{J})$ pump laser beam which was scanned across the absorption region while the less intense $(20-100 \mu \mathrm{J})$ probe laser beam was delayed in time and 
fixed at a frequency corresponding to the maxima of the most intense bands on the FES. The intensity of the pump laser that we used was determined as to be low enough to limit saturation effects but still high enough to obtain distinguishable spectral holes.

Inside the second chamber the anticollinear excitation and ionisation laser beams cross the molecular beam in the source of the time-of-flight mass spectrometer. Resonant enhanced two-photon two-colour ionisation (2C-R2PI) associated with time-of-flight mass spectrometry enables the recording of mass selected excitation spectra. Ionised clusters dissociate very efficiently depending on the energy of the ionising photon. The use of time-of-flight mass spectrometer enables to distinguish the metastable ions coming from slow dissociation $\left(10^{4} \mathrm{~s}^{-1}<k_{\text {diss }}<10^{6} \mathrm{~s}^{-1}\right)$ since their temporal shapes are more or less strongly asymmetric towards the longer times as compared to that of the non-dissociating or rapidly dissociating ions. The absorption spectra of the species in which dissociation takes place is obtained by temporally selecting the metastable ion signal and by monitoring its variation as a function of the excitation wavelength.

\section{Ionisation of photoexcited AD van der Waals complexes}

When the fluorescence yield and ionisation efficiency do not depend on the energy of the (AD)* state, both FES and MSES spectra are expected to be identical with the absorption spectrum of $\mathrm{AD}$. This is not the case for the complexes under study: the electron transfer $A^{*} D \sim A^{-} D^{+}$takes place either for all levels of the excited complex including the lowest ones (E-isomers) or for the levels above the electron-transfer threshold (R-isomers). Accordingly, different FES spectra are obtained depending whether the resonant or exciplex fluorescence is monitored. Concerning the ionisation step, two different processes corresponding in the zero-order approximation to:
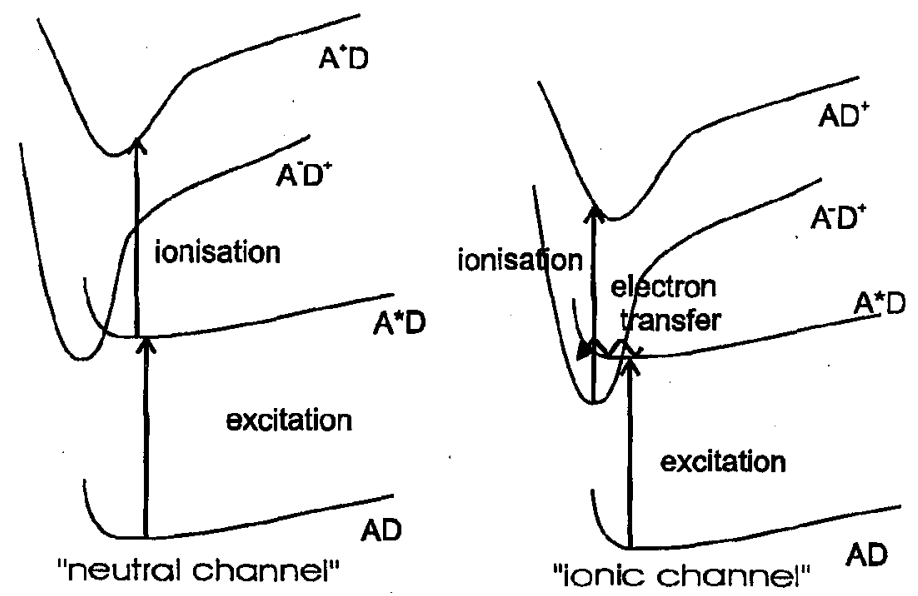

Fig. 2. Description of the two ionisation channels involved in the ionisation process of photo-excited AD complexes. 
(i) ionisation of the excited acceptor: $\mathrm{A}^{*} \mathrm{D}+h \nu_{\text {ion }}=\mathrm{A}^{+} \mathrm{D}$,

(ii) photodetachment from $\mathrm{A}^{-}$ion: $\mathrm{A}^{-} \mathrm{D}^{+}+h \nu_{\mathrm{ion}}=\mathrm{AD}^{+}$,

which may differ by their ionisation cross-sections have also to be considered (Fig. 2).

To illustrate this point, we report here MSES of the anthracene-dimethyl-o-toluidine (An-DMOT) complex. The reason of this choice is a large number of isomers (6) as evidenced by hole burning spectroscopy [1].

\subsection{Results}

The An-DMOT MSES is presented in Fig. $3 \mathrm{c}$ together with the corresponding R-FES (Fig. 3a) and E-FES (Fig. 3b). Strong differences are clearly seen:

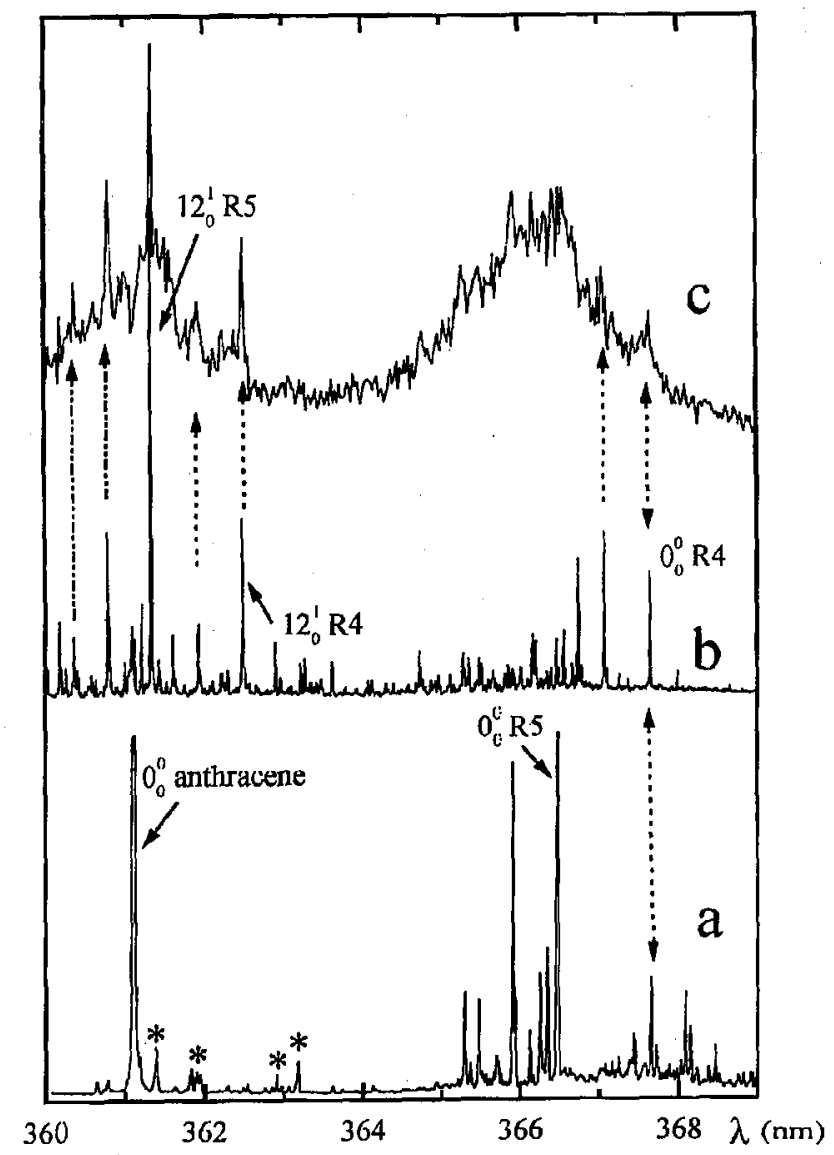

Fig. 3. Fluorescence excitation spectra of the anthracene-dimethyl-o-toluidine complex with detection: (a) of the resonant fluorescence ( $375 \mathrm{~nm}$ domain), the free anthracene "hot" bands are marked by $*$, (b) of the exciplex fluorescence (450 $\mathrm{nm}$ domain) and (c) mass selected low resolution excitation spectrum (the ionisation wavelength was fixed at $310 \mathrm{~nm} \approx 4.0 \mathrm{eV}$ ). 
(i) two broad $0_{0}^{0}$ and $12_{0}^{1}$ bands of the E-type isomer strong in the MSES are practically absent from the E-FES (they can only be observed by using a much higher detection sensitivity).

(ii) the bands corresponding to the $0_{0}^{0}$ narrow band system of the R5 isomer observed in the R-FES are absent from the MSES while those corresponding to the $12 \frac{1}{0}$ system only observed in the E-FES spectrum are present with an intensity distribution similar to that observed in the MSES.

(iii) the $0_{0}^{\circ}$ band of the R4 isomer observed in both the R-FES and E-FES appears in the MSES with a relatively low intensity while the corresponding band of the $12_{0}^{1}$ system is much stronger.

(iv) the bands assigned to R1, R2 and R3 isomers, very weak in R-FES are not observed in MSES.

The MSES of An-DMA and An-DEA complexes, not shown here, present the features corresponding to the $\mathrm{E}$ isomer while the narrow bands of the $\mathrm{R}$ isomers are absent.

\subsection{Discussion}

These results may be accounted for if assumed that the ionisation efficiency is much higher for complexes in the $\mathrm{A}^{-} \mathrm{D}^{+}$as compared to the $\mathrm{A}^{*} \mathrm{D}$ state:

(1) In E-type isomers the high electron transfer rate $\left(k_{\mathrm{ET}}>10^{12} \mathrm{~s}^{-1}\right)$ implies that the excited state which absorbs the ionising photon is not the initially prepared $\mathrm{A}^{*} \mathrm{D}$ state but the ionic state $\mathrm{A}^{-} \mathrm{D}^{+}$which is efficiently ionised.

(2) The excitation of the lowest vibronic transitions of the $0_{0}^{0}$ system of the R5 isomer correlating with the anthracene $0_{0}^{0}$ band results in a resonant fluorescence which shows that the $A^{*} D \leadsto A^{-} D^{+}$transition does not occur during its lifetime. The ionization of this state is not efficient. In contrast to it, the $A^{*} D \sim A^{-} D^{+}$ transition takes place upon the $12_{0}^{1}$ excitation at the time scale much shorter than the laser pulse duration, so that the ionising photon is absorbed from the efficiently ionised $\mathrm{A}^{-} \mathrm{D}^{+}$state.

(3) The R4 isomer shows a particular behaviour: the dual (resonant + exciplex) fluorescence is emitted from the origin $0_{0}^{0}$ band (while emission of exciplex fluorescence only is observed for all other levels) showing that the electron transfer rate is of the same order of magnitude as the fluorescence rate of the $A^{*} D$ state. One can thus consider that the ionising photon interacts with an $A^{*} D / A^{-} D^{+}$ "mixture" while in the case of the $12{ }_{0}^{1}$ excitation all complexes are in the $\mathrm{A}^{-} \mathrm{D}^{+}$ state. This explains a lower intensity of the R4 isomer origin band than for the corresponding $12 \frac{1}{0}$ band. From the intensity of these bands in the MSES we have determined that the ionisation efficiency is one order of magnitude higher for complexes in $\mathrm{A}^{-} \mathrm{D}^{+}$state than for complexes in $\mathrm{A}^{*} \mathrm{D}$ states.

In conclusion, this ionisation study on the An-DMOT complex has shown that the efficiency of the photo-detachment from the $\mathrm{A}^{-} \mathrm{D}^{+}$state is (at least by one order of magnitude) larger than that of the ionisation of the neutral $A^{*} D$ state. The reason of this difference is not obvious and we do not know whether we are dealing with a general rule or with a specific effect due to differences between oscillator strengths of transitions from bound state to the ionisation continua (or 
to auto-ionised states). The fact that a similar behaviour was previously observed for so different systems as $\mathrm{Na}^{+} \mathrm{I}^{-} \leftrightarrow \mathrm{NaI}^{*}[9]$ or naphthalene trimer [3] argues in favour of a general rule.

\section{Excimer formation from photoexcited anthracene dimers and clusters}

The aromatic hydrocarbons form in excited state stable dimers (excimers) similar to exciplexes. Their stability is due to excitonic and charge resonance interactions between two sets of diabatic excited states, the locally excited states $\mathrm{A}^{*} \mathrm{~A}$ and the charge transfer states $\mathrm{A}^{-} \mathrm{A}^{+}$. The adiabatic potential energy surface of the dimer excited state results from the configuration interaction between these zero-order states [5].

The excimer emission of jet cooled anthracene clusters was first evidenced by Lim et al. [7] who postulated the existence of two isomers for the dimer. Since in this work, selective diagnoses like 2C-R2PI or hole burning spectroscopy were absent, we decided to use such means to study anthracene dimers and larger clusters in order to unambiguously assign the dimer spectral features.

\subsection{Results \\ 4.1.1. Fluorescence and hole burning spectroscopy}

The excited anthracene dimer and clusters emit only excimer type fluorescence centred at $450 \mathrm{~nm}$ when scanning the excitation laser between 365 and $390 \mathrm{~nm}$. The E-FES obtained by selectively detecting this excimer emission is composed of three broad bands called $A, B$, and $C$, the central one $B$ being structured as shown in Fig. 4a. The fluorescence spectrum does not depend on the excitation wavelength but the decay time varies with $\lambda_{\text {exc }}$ and is respectively of $200 \mathrm{~ns}, 11 \mathrm{~ns}$, and $40 \mathrm{~ns}$ upon excitation at $367.8 \mathrm{~nm}, 375.4 \mathrm{~nm}, 381 \mathrm{~nm}$ which corresponds to the $A, B, C$ bands maxima. The differences in both lifetimes and spectral shapes strongly suggest the presence of different ground state species which can be checked by using the hole burning spectroscopy. The hole burning spectra (HBS) obtained by fixing the probe laser on the maxima of $A, B, C$ are shown respectively in Figs. $4 \mathrm{~b}-\mathrm{d}$. They are all different which demonstrates that $A, B, C$ bands are due to different ground state species.

The HBS obtained with the probe laser fixed at the maximum of the $A$ band (Fig. 4c) shows one broad band only similar to that observed in the E-FES. The shift of the maximum relative to the anthracene origin band position is $\delta \nu=-507 \mathrm{~cm}^{-1}$.

The HBS recorded with the probe laser fixed at the maximum of the $B$ band (Fig. 4b) reproduces the resolved pattern observed in the excimer fluorescence excitation spectrum (E-FES) but shows a second band overlapping the $A$ band. The width of the origin sharp band at $26638 \mathrm{~cm}^{-1}$ is $4 \mathrm{~cm}^{-1}$, significantly larger than the typical 0.8 to $1.2 \mathrm{~cm}^{-1}$ width expected for the rotational contour as determined from our study of the AD complexes [1]. This width can be attributed to a homogeneous broadening due to a nonradiative process with $k=0.7 \times 10^{12} \mathrm{~s}^{-1}$. The sharp bands blue shifted by respectively 390 and $780 \mathrm{~cm}^{-1}$ from the sharp origin band are assigned to the $12_{0}^{1}$ and $12_{0}^{2}$ vibronic transitions of anthracene. 


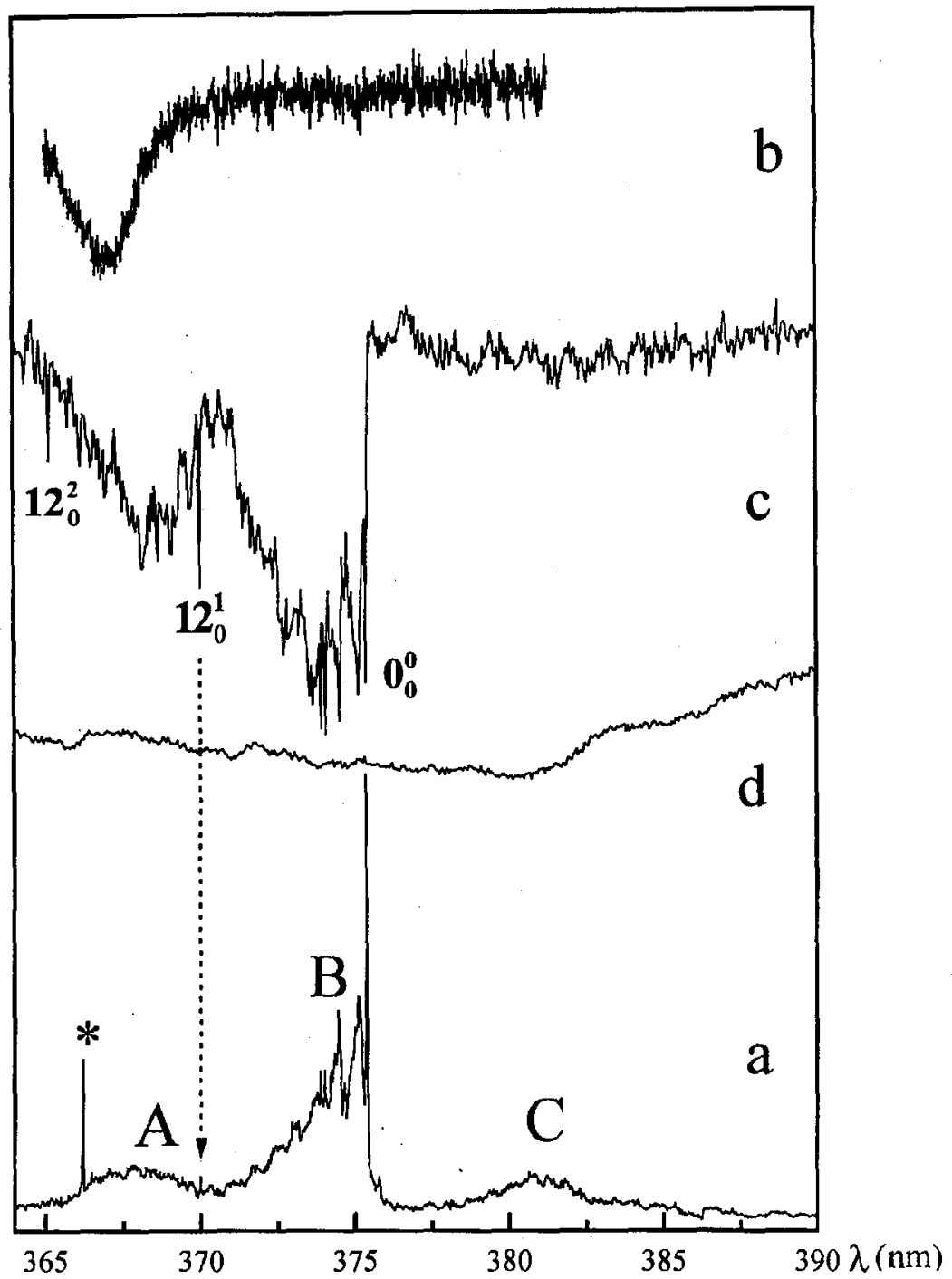

Fig. 4. (a) Fluorescence excitation spectra of the anthracene clusters with detection of the exciplex fluorescence ( $450 \mathrm{~nm}$ domain) characterised by three broad bands labelled $A, B, C$, a free anthracene "hot" band is marked by *, and hole burning spectra with probe wavelength fixed respectively (b) on the maximum of the $A$ broad band $(367.8 \mathrm{~nm})$, (c) on the maximum of the $B$ broad band $(375.4 \mathrm{~nm})$, and (d) on the maximum of the $C$ broad band $(382 \mathrm{~nm})$. The (b) spectrum was obtained with a delay of $\approx 1 \mu$ s between pump and probe laser pulses due to the long lifetime of the excimer emission in the case of the $A$ band. Therefore due to the speed of the molecules in the He expansion $(1760 \mathrm{~m} / \mathrm{s})$ it was necessary to spatially shift the probe laser beam downstream by ca. $1.2 \mathrm{~mm}$ from the pump laser beam in order that pump and probe laser beams interact with the same population. 
The $12_{0}^{1}$ band is also present on the E-FES but with a very weak intensity while the $12_{0}^{2}$ is absent. The low intensity of the $12_{0}^{1}$ and the absence of the $12_{0}^{2}$ bands in the E-FES indicates that the $B$ species undergo in the excited state an energy dependent nonradiative process competing with either excimer formation or excimer fluorescence.

The HBS (Fig. 4d) obtained by probing the $C$ band $\left(\delta \nu=-1450 \mathrm{~cm}^{-1}\right)$ covers the whole spectral domain $(25 \mathrm{~nm})$ with a maximum located at $381 \mathrm{~nm}$.

\subsubsection{Mass selective ionisation spectroscopy}

MSES of anthracene dimers and clusters were obtained using 2C-R2PI associated to time-of-flight mass spectrometry.

(1) Dimer:

The anthracene dimer MSES obtained by setting the ionisation wavelength to $310 \mathrm{~nm}$ exhibits two broad bands in the $360-370 \mathrm{~nm}$ spectral domain (Fig. $5 \mathrm{~b}$ ), located at 367.7 and $361 \mathrm{~nm}$, and separated by $350 \mathrm{~cm}^{-1}$. This shift is slightly lower than the frequency of the $\nu_{12}$ intramolecular mode of anthracene. The origin broad band peaks at $367.7 \mathrm{~nm}$ and corresponds to the $A$ band observed in E-FES (Fig. 5a). It should be noted that this spectrum was obtained in conditions such

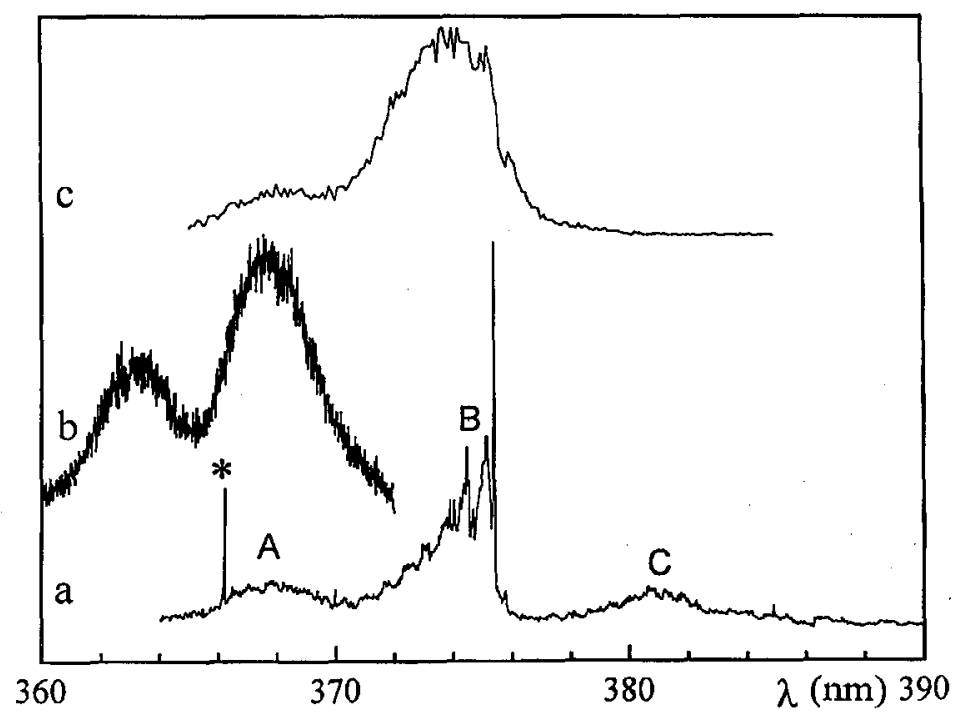

Fig. 5. (a) Fluorescence excitation spectra of the anthracene clusters with detection of the excimer fluorescence (450 nm domain), and mass selected excitation spectra obtained by monitoring as a function of the excitation wavelength, (b) the variation of the ion signal corresponding to the dimer mass, and (c) the variation of the ion signal corresponding to the trimer mass (the ionisation wavelength was fixed at $310 \mathrm{~nm}$ for both spectra). The absence of a dye laser covering the 360-390 $\mathrm{nm}$ spectral range required the use of two dyes: DMQ for the $360-372 \mathrm{~nm}$ domain and BBQ for the $365-390 \mathrm{~nm}$ domain. For the sake of clarity we only present here the dimer MSES in the 360-372 nm domain. 
that the dimer ion signal is free from fragments arising from dissociation of higher clusters. Otherwise, a weak signal, almost continuous, was observed further to the red and can be attributed to the dissociation of higher clusters, as deduced from MSES spectra obtained for anthracene trimer, tetramer, pentamer.

(2) Trimer:

The absorption spectrum of the trimer as deduced from its MSES (Fig. 5c) is similar to the HBS of the $B$ band species (Fig. 4c). However in the MSES, the sharp origin band as well as the other bands contributing to the structure of the $B$ band are less apparent probably because of the weakness of the ion signal. This can be related to the short lifetime of the excited state and its low ionisation efficiency, resulting in a weak signal to noise ratio. The red shift of the trimer maximum relative to the anthracene origin band is $940 \mathrm{~cm}^{-1}$, slightly lower $\left(\approx 70 \mathrm{~cm}^{-1}\right)$ than twice that of the dimer. When the trimer species is excited at the absorption maximum ( $\lambda \approx 375 \mathrm{~nm}$ ), the mass spectrum shows in addition to the trimer ions, metastable dimer ions. We have simultaneously recorded the trimer ion signal and the metastable part of the dimer ion signal as a function of the excitation wavelength. Both MSES are similar in shape which indicates that the trimer ion efficiently dissociates to form a dimer. As a similar process is absent in the case of the dimer, it indicates that the trimer ion stability is much lower than that of the dimer. Furthermore, the trimer ion signal shows a rapid decrease when increasing the delay between the excitation and ionisation lasers, which indicates that the trimer excited state has a short lifetime, of the same order of magnitude as the fluorescence lifetime measured for the $B$ band.

(3) Clusters:

The MSES of the anthracene clusters $\left(\operatorname{An}_{n}\right.$ with $\left.3<n<7\right)$ show that their absorption spectrum is very broad, unstructured, and strongly red shifted $\left(\delta \nu=-1300 \mathrm{~cm}^{-1}\right.$ for the tetramer) which suggests that the HBS corresponding to the $C$ band of the MSES results from the overlapping of the individual absorption spectra of anthracene clusters. This is confirmed by (i) a larger red shift of their band maximum compared to that of the dimer and (ii) a strong dependence of the intensity of this band in the E-FES on the anthracene reservoir temperature and on the delay between the gas and laser pulses. Slightly metastable dimer ion signal is observed upon excitation at $381 \mathrm{~nm}$ indicating that these clusters dissociate more rapidly than the trimer species.

\subsection{Discussion}

In this work we have been able to assign unambiguously the $A$ band to an anthracene dimer. The $B$ band was previously attributed by Lim [7] to an isomeric form of the dimer on the basis on fluorescence excitation spectra and temperature effects. From the present data the $B$ band is more likely to be due to an anthracene trimer. This species exhibits however a peculiar behaviour when compared to the dimer:

(i) the $B$ band is much more intense than the $A$ band in the FES spectrum although the relative concentration of trimer is expected to be smaller than that of the dimer, 
(ii) the excimer type fluorescence decay is much shorter for the excitation of the $B$ band than for the $A$ band,

(iii) the $B$ band displays a resolved structure while the $A$ band is broad.

These observations seem to indicate a short radiative lifetime for the excited trimer and may be rationalised by considering that the optically excited state bears more locally excited state character than that corresponding to the dimer. These differences are probably related to the structure of the ground state cluster. While the ground state dimer may present the favourable geometry needed to achieve excimer formation, the trimer geometry seems to give rise in the excited state to a species that can be viewed as an anthracene monomer perturbed by the presence of the two others. Such a behaviour has also been observed in naphthalene clusters by Lim et al. [3], where the excited state of the trimer possesses more locally excited state character (structured absorption spectrum, barrier for excimer formation) than the dimer (broad absorption spectrum, no barrier for excimer emission).

In conclusion, this work on anthracene clusters enables to evidence (i) that all clusters emit only excimer type fluorescence which indicates that the electron transfer rate is high enough $\left(k_{\mathrm{ET}}>10^{9} \mathrm{~s}^{-1}\right)$ to totally quench the resonant fluorescence, (ii) that the dimer and trimer exhibit strongly different absorption properties and dynamics and (iii) that the absorption of anthracene clusters $\mathrm{An}_{n}$ (with $n>3$ ) is very broad and overlaps with dimer and trimer absorption and the lifetime of their excited state is intermediate between that of the trimer and that of the dimer.

\section{Electron transfer in AD complexes bonded by a hydrogen bond}

We present here the results of our study of the model-system for hydrogen-bonded TFAE/aniline-derivatives complexes, TFAE-DMA. In these systems, the geometry imposed by the $\mathrm{H}$ bond can modify the efficiency of electron transfer by orienting the non-bonding pair of the $\mathrm{N}$ atom more or less parallel or perpendicular to the plane of the anthracene ring [10]. Besides this geometrical factor, electronic factors related to the $\mathrm{H}$ bond may also play a role in the dynamics of the excited state: the lone pair located on the nitrogen atom is expected to be less accessible when involved in a hydrogen bond.

\subsection{Bare molecule}

The excitation spectrum of TFAE is presented in Fig. $6 \mathrm{a}$. The $0_{0}^{0}$ transition is located at $26876 \mathrm{~cm}^{-1}$, and is red-shifted by $811 \mathrm{~cm}^{-1}$ relative to that of unsubstituted anthracene. This shift is slightly more important than for methylanthracene $\left(748 \mathrm{~cm}^{-1}\right)$. It is followed by a three-member progression built on a $46 \mathrm{~cm}^{-1}$ mode assigned to a torsion of the substituent. The modes which correlate to the $\overline{11} b_{1 \mathrm{~g}}$ and $12 a_{1 \mathrm{~g}}$ vibrations of the anthracene ring are located at 218 and $390 \mathrm{~cm}^{-1}$, respectively [11]. Much weaker bands appear at $129 \mathrm{~cm}^{-1}$ in the red of the $0_{0}^{0}$ band, with the same vibrational pattern: hole burning experiments have shown that they are due to other ground-state species, probably a torsion isomer of the molecule (Figs. 6b and c). This result contrasts with the properties of a previously studied chiral naphthalene derivative, that bears similar substituents, 


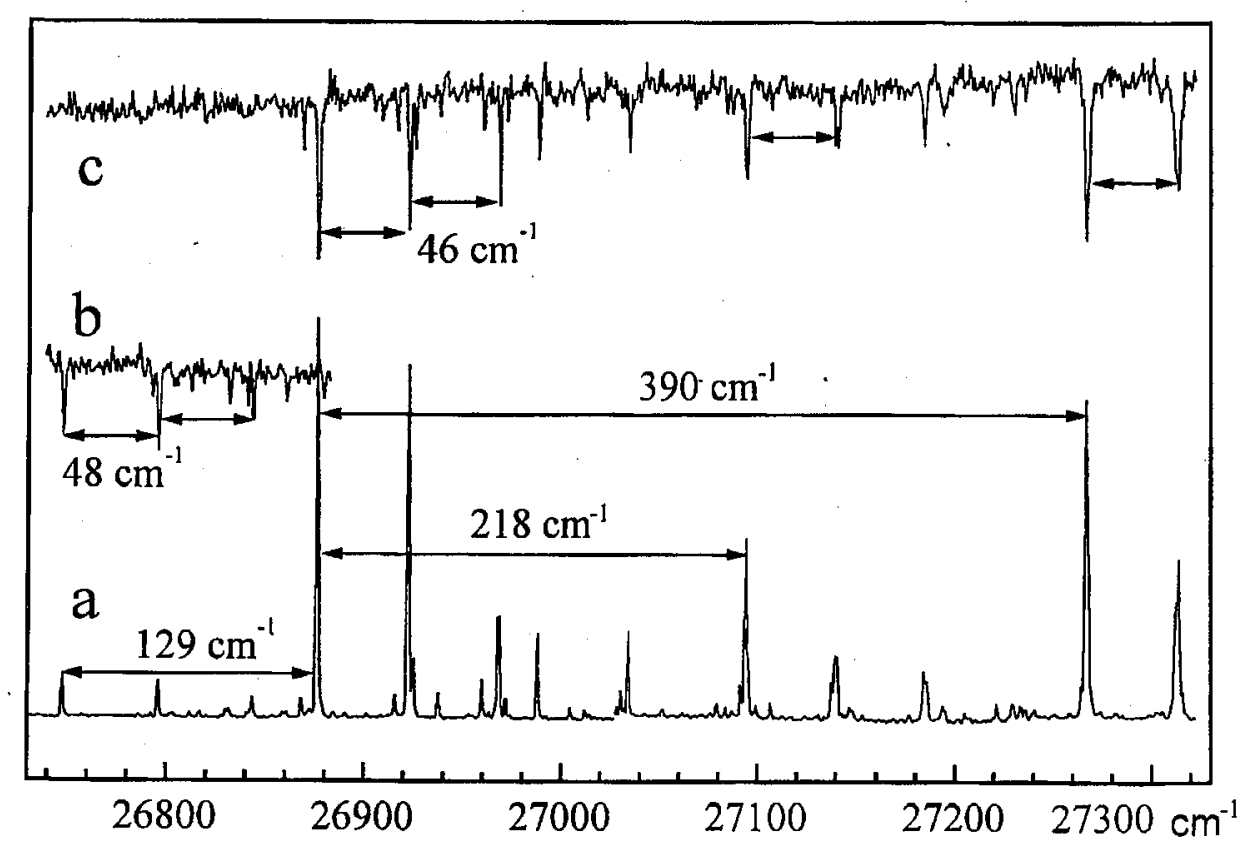

Fig. 6. (a) Fluorescence excitation spectrum of 2,2,2-trifluoro-1-(9-anthryl)ethanol with detection of the resonant fluorescence ( $375 \mathrm{~nm}$ domain), and hole burning spectra of TFAE with (b) the probe laser fixed at $26747 \mathrm{~cm}^{-1}$ and (c) the probe laser fixed at $26876 \mathrm{~cm}^{-1}$.

and that has been shown to exist under a single isomeric form under supersonic-jet conditions [12]. These differences in behaviour may be related to a larger steric hindrance in the case of an anthracene ring. The lifetime of the $0_{0}^{0}$ level is of $15 \mathrm{~ns}$.

\subsection{TFAE-DMA complex}

The R-FES and E-FES are shown in Figs. 7a,b, respectively, while the HBS recorded with the probe fixed at $26613 \mathrm{~cm}^{-1}$ is presented in Fig. 8 . This HBS shows unambiguously that only one isomer with a structured spectrum is formed in the jet. Besides this structured spectrum, a broad band appears weakly in E-FES and has been assigned to an E-type isomer with similar properties as those observed for the $\mathrm{E}$ isomer of the An-DMA complex. This broad band is also observed in MSES, in contrast to the structured spectrum. This can be explained by the same reasons as proposed before for An-DMOT. The $0_{0}^{0}$ band of the structured complex is located at $26571 \mathrm{~cm}^{-1}$, and it is red-shifted by $305 \mathrm{~cm}^{-1}$ relative to the bare molecule. This red shift is relatively small when compared to the red shift observed for the An-DMA complex $\left(515 \mathrm{~cm}^{-1}\right)$. A possible explanation is that this smaller red-shift is related to a decrease in the dispersion forces resulting from a smaller overlap between the aromatic rings as consequence of the geometry imposed by the $\mathrm{H}$ bond. 


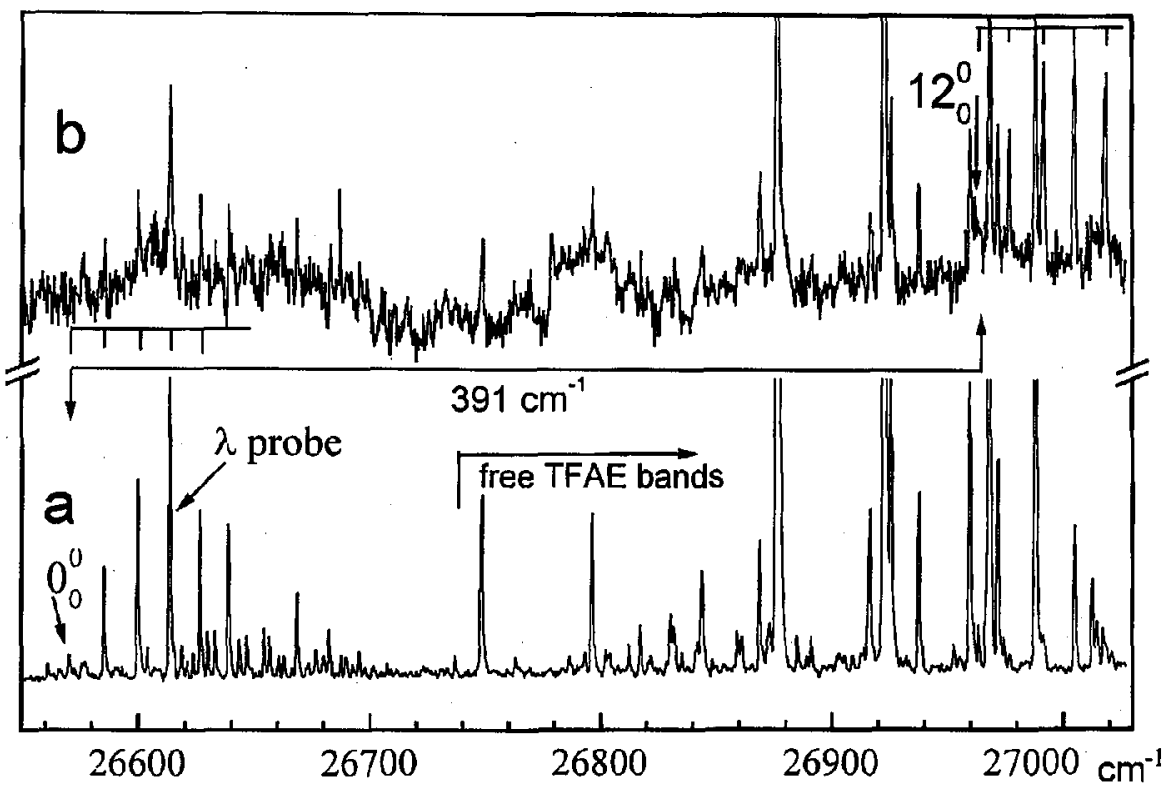

Fig. 7. Fluorescence excitation spectrum of 2,2,2-trifluoro-1-(9-anthryl)ethanol-dimethylaniline complex: (a) detection of the resonant fluorescence (375 nm domain). The signal has been recorded in a $30 \mathrm{~ns}$ temporal gate. (b) Detection of the exciplex fluorescence (450 nm domain). The signal has been recorded in a $100 \mathrm{~ns}$ temporal gate delayed by $40 \mathrm{~ns}$.

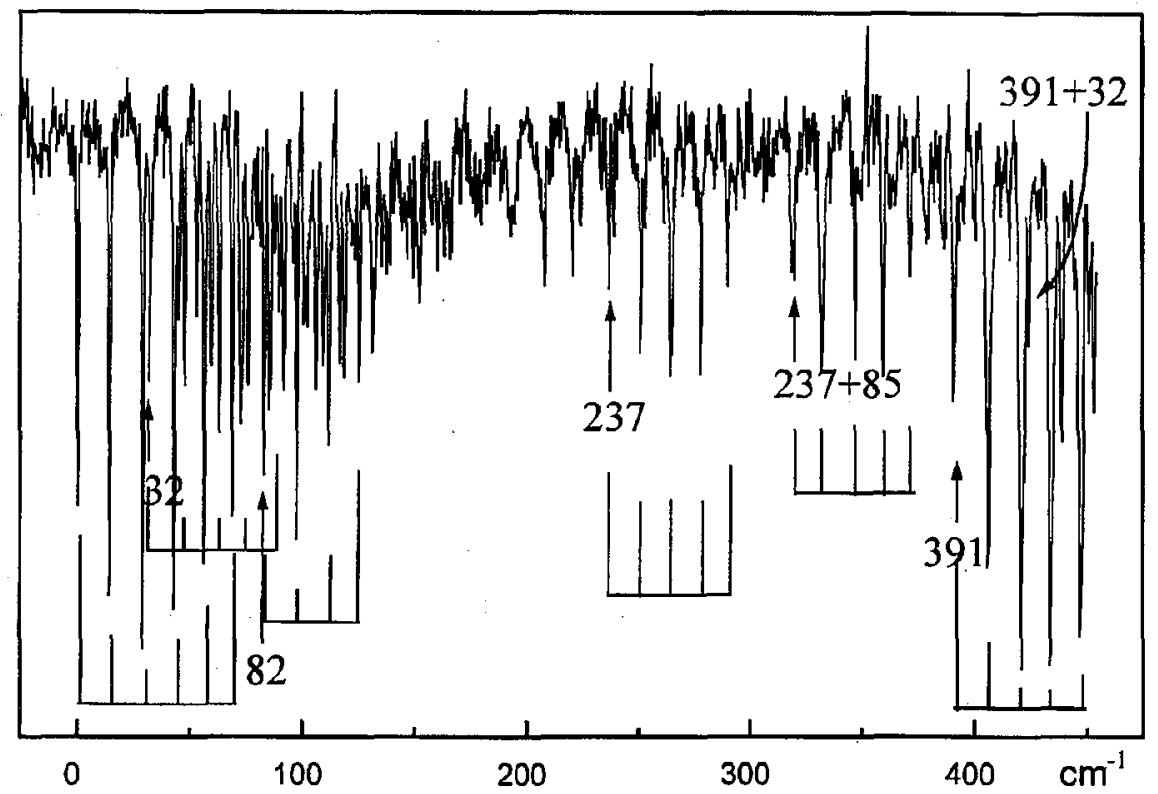

Fig. 8. Hole burning spectrum of the TFAE-DMA complex. The probe laser is fixed at $26613 \mathrm{~cm}^{-1}$ (most intense band of the $0_{0}^{0}$ narrow band system). 


\subsubsection{Vibrational structure}

The absorption spectrum of TFAE-DMA shows a complex vibrational structure built on an intermolecular mode of $14 \mathrm{~cm}^{-1}$. This mode is also observed in combination with other van der Waals modes of $32 \mathrm{~cm}^{-1}$ and $82 \mathrm{~cm}^{-1}$, and in combination with the intramolecular $218 \mathrm{~cm}^{-1}$ and $390 \mathrm{~cm}^{-1}$ modes. The $14 \mathrm{~cm}^{-1}$ mode might be assigned to a slipping motion between the anthracene ring and the aniline derivative: such low frequency modes have also been observed $\left(19 \mathrm{~cm}^{-1}\right)$ in the An-DMA complex [1]. The most striking difference is the distribution of the Franck-Condon factors that clearly differs for the An-DMA and TFAE-DMA complexes: whereas the An-DMA exhibits a strong $0_{0}^{0}$ band followed by weaker transitions, the TFAE-DMA displays a vibrational structure whose maximum intensity is located on the third or fourth member of the progression. This shows that the shape of the excited state potential energy surface is more modified along this coordinate upon excitation for the TFAE-DMA complex than for the An-DMA complex. The $82 \mathrm{~cm}^{-1}$ frequency may be assigned to a stretching motion of the $\mathrm{H}$ bond, because it has not been observed in An-DMA. Here again, it is observed in combination with the $11 b_{1 \mathrm{~g}}$ and $12 a_{1 \mathrm{~g}}$ modes.

\subsubsection{Energy dependence of the exciplex formation efficiency}

The ratio between the resonance and the exciplex emission intensities $I_{\mathrm{E}} / I_{\mathrm{R}}$ strongly depends on the excitation wavelength. No exciplex emission is observed when exciting the narrow bands of the complex that correlate with the $0_{0}^{0}$ band of TFAE. The bands observed in this region in Fig. $7 \mathrm{~b}$ are due to the intense resonant fluorescence insufficiently discriminated by our broad-band band-pass monochromator. The $I_{\mathrm{E}} / I_{\mathrm{R}}$ ratio strongly increases when the $391 \mathrm{~cm}^{-1}$ intramolecular mode of TFAE is excited, but a dual fluorescence is still detected in this excitation energy range. This result is in contrast with the An-DMA complex for which no resonant fluorescence is observed at this excess energy. Therefore, the main difference between An-DMA and TFAE-DMA is a higher threshold for exciplex formation in the latter system. It is to be noted that this result cannot be inferred from the thermodynamical data of the bare molecules: the donor (DMA) is the same in both complexes, while the electron affinity of the acceptor is expected to be higher for TFAE thanks to the stabilising effect of the electron-withdrawing substituent. On this basis, electron transfer should be easier in TFAE-DMA, which is obviously not observed. The increase in the threshold for electron transfer is more likely related to the $\mathrm{H}$ bond that increases the ionisation potential of the amine molecule and constraints the geometry of the complex. Hydrogen bonding thus results in a decrease in the electron transfer efficiency as observed in solution for intramolecular exciplex formation in tetrahydroisoquinoline [10]. The same conclusion has been drawn for rigidly-linked naphthalene-trialkylamine compounds in alkanol solutions [13] for which the lifetime of the locally excited state has been shown to be by far longer than in nonprotic solvents of similar polarity. The pronounced dependence of the charge transfer rate upon temperature has been interpreted in terms of a large barrier for electron transfer, which in turn has been related to a concerted process in which the breakage of the $\mathrm{H}$ bond occurs simultaneously with the electron transfer. 
In conclusion, substitution in 9 position of anthracene by a hydrogen donating group slightly modifies the spectroscopic properties of the complex with DMA. In both anthracene and TFAE complexes, one isomer only with a structured excitation spectrum is observed. The TFAE-DMA complex differs by the presence of long progressions built on a low-frequency $14 \mathrm{~cm}^{-1}$ mode, which shows a strong modification of the potential energy surface, probably along a coordinate involving a slipping motion between the aromatic rings, and a $82 \mathrm{~cm}^{-1}$ mode assigned to the stretching of the $\mathrm{H}$ bond. The photoinduced electron transfer is observed for both systems, but takes place with a higher threshold in the hydrogen bonded system. This could not be expected from the thermodynamical data of the isolated systems (ionisation potential of the DMA donor, electron affinity of the acceptor) and has thus been related to the presence of the $\mathrm{H}$ bond.

\section{Acknowledgments}

We acknowledge the technical help of Benjamin Tardivel and Alexandre Biasi in running the experiments.

\section{References}

[1] (a) V. Brenner, Ph. Millié, F. Piuzzi, A. Tramer, J. Chem. Soc. Faraday Trans. 93, 3277 (1997); (b) A. Tramer, V. Brenner, Ph. Millié, F. Piuzzi, J. Phys. Chem. 102, 2798 (1998).

[2] F. Piuzzi, Chem. Phys. Lett. 209, 484 (1993).

[3] H. Saigusa, E.C. Lim, J. Phys. Chem. 99, 15738 (1995).

[4] H. Saigusa, S. Sun, E.C. Lim, J. Phys. Chem. 96, 2083 (1992).

[5] W.T. Yip, D.H. Levy, J. Phys. Chem. 100, 11539 (1996).

[6] T. Chakraborty, E.C. Lim, Chem. Phys. Lett. 207, 99 (1993).

[7] T. Chakraborty, E.C. Lim, J. Phys. Chem. 97, 11151 (1993).

[8] W.H. Pirkle, D.W. House, J. Org. Chem. 44, 1957 (1979).

[9] C. Jouvet, S. Martrenchard-Barra, D. Solgadi, C. Dedonder-Lardeux, M. Mons, G. Gregoire, I. Dimicoli, F. Piuzzi, J.P. Visticot, J.M. Mestdagh, P. d'Olivera, P. Meynadier, M. Perdrix, J. Phys. Chem. A 101, 2555 (1997).

[10] P.K. Bera, A. Chakraborty, M. Chowdury, Chem. Phys. Lett. 277, 57 (1997).

[11] W.R. Lambert, P.M. Felker, J.A. Syage, A.H. Zewail, J. Chem. Phys. 81, 2195 (1984).

[12] K. Le Barbu, V. Brenner, Ph. Millié, F. Lahmani, A. Zehnacker-Rentien, J. Phys. Chem. A 102, 128 (1998).

[13] A.M. Brun, A. Harriman, Y. Tsuboi, T. Okada, N. Mataga, J. Chem. Soc. Faraday Trans. 91, 4047 (1995). 